\title{
A Matter of TACEte: Plain Vanilla or Combination?
}

\author{
David E. Kaplan ${ }^{1,2}$
}

Published online: 27 August 2019

(c) This is a U.S. Government work and not under copyright protection in the US; foreign copyright protection may apply 2019

$\begin{array}{ll}\text { Abbreviations } \\ \text { AFP } & \text { Alphafetoprotein } \\ \text { BCLC } & \text { Barcelona clinic liver cancer } \\ \text { BMI } & \text { Body-mass index } \\ \text { DFS } & \text { Disease-free survival } \\ \text { HBV } & \text { Hepatitis B infection } \\ \text { HCC } & \text { Hepatocellular carcinoma } \\ \text { ITT } & \text { Intention-to-treat } \\ \text { IPTW } & \text { Inverse probabilty of treatment weighting } \\ \text { mRECIST } & \text { Modified response evaluation criteria in solid } \\ & \text { tumors } \\ \text { OS } & \text { Overall survival } \\ \text { PA } & \text { Percutaneous ablation } \\ \text { PFS } & \text { Progression-free survival } \\ \text { RCT } & \text { Randomized controlled trial } \\ \text { RFA } & \text { Radiofrequency ablation } \\ \text { TACE } & \text { Transarterial chemoembolization } \\ \text { TE } & \text { Transarterial embolotherapy }\end{array}$

Take Home Points

- Transarterial embolotherapy (TE) using lipiodol \pm cytotoxic chemotherapy and embolic agents or drug-eluting beads, bland embolization, or radioembolization are frequently employed for the treatment of unresectable HCC but rarely achieve sterilization of the tumor bed.

- To improve local control, many centers have empirically combined percutaneous, laparoscopic or radiotherapy ablation with TE.

- Nevertheless, these combinations have not been shown in randomized controlled trials to improve relevant outcomes.

David E. Kaplan

dakaplan@pennmedicine.upenn.edu

1 Division of Gastroenterology and Hepatology, Perelman School of Medicine, University of Pennsylvania, 3400 Civic Center Drive, PCAM 7S GI, Philadelphia, PA 19104, USA

2 Corporal Michael J. Crescenz VA Medical Center, Philadelphia, PA 19104, USA
- The present study attempts to demonstrate using causal inference methodology with retrospective data that a combination of transarterial chemoembolization (TACE) and radiofrequency ablation provides superior tumor control relative to TACE alone.

Hepatocellular carcinoma (HCC) continues to be the second-leading cause of cancer death worldwide. Only a small minority of patients with HCC have resectable disease at diagnosis, either due to anatomical or clinical considerations such as concomitant portal hypertension, decompensated liver disease, or comorbid diseases [1]. Initial treatment for the majority of patients who at diagnosis have unresectable early-intermediate stage disease consists of loco-regional therapy including percutaneous or laparoscopic tumor ablation, transarterial therapy, or conformal radiotherapy. Within these three broad categories, multiple specific variations of the approaches exist. Few high-quality data exist to inform practitioners which subtype (e.g. superselective transarterial chemoembolization with drug-eluting beads vs. iodized oil and gelfoam) to recommend to patients. Still fewer highquality data guide potential combinations of the distinct approaches.

Ablation of the tumor bed, most commonly with probedelivered microwave or radiofrequency ablation (RFA), but increasingly through targeted radiotherapy, achieves a high rate of local control for small $(\leq 3 \mathrm{~cm})$ HCCs [2]. Challenges to tumor sterilization for percutaneous ablation (PA) are either technical or biological. Technical challenges include the limited volume of effective ablative energy, tumor location, with risk of a heat-sink effect for tumors adjacent to large blood vessels, risk of capsular injury with subcapsular tumors, and risk of injury to the lung, diaphragm or heart with high dome tumors, as well as tumor localization given the reliance on non-contrast-enhanced imaging modalities to guide probe placement. Biological challenges to percutaneous ablation include tumor multifocality, satellites, and microscopic invasion that may extend outside the delivered treatment zone. While ablative therapies may sterilize the tumor bed with low rates of serious hepatic injury due to 
the localized effects, recurrence rates at 1-year approximate $10-15 \%$ [3].

Transarterial embolotherapy (TE) using lipiodol \pm cytotoxic chemotherapy and embolic agents or drug-eluting beads, bland embolization, or radioembolization, can be more flexibly applied from an anatomic perspective and be applied to larger tumor sizes. Nevertheless, TE rarely sterilizes any tumor bed with residual viable tumor identified in $>80 \%$ of cases [4]. No single prospective randomized controlled study has ever proved the efficacy of TACE for intermediate stage HCC, with the strongest data that support its use generated from meta-analysis [5]. Nonetheless, embolization-based therapies are generally considered standard-of-care for bridging or palliation for early-intermediate stage HCC, particularly for tumors $>3 \mathrm{~cm}$ in diameter and/or tumors located in unfavorable anatomic locations for percutaneous ablation.

TACE and PA a priori appear naturally complementary. The use of lipiodol in embolotherapy to "paint" tumors would be expected to improve localization with non-contrast imaging and to improve estimation of tumor volume in order to optimize the application of ablative energy. TACE might also reduce heat-sink effects of the peritumoral vasculature in order to increase the effective volume of ablation. As a complement to TACE, PA might reduce the volume of residual viable tumor in the embolization zone, theoretically improving local control rates and retarding tumor recurrence. Such complementation without unacceptable increases in adverse effect rates, if convincingly demonstrated, would likely shift the standard-of-care.

Yet, strong evidence of this complementation effect of TE on PA or of PA on TE in unresectable HCC has been slow to evolve. Challenges in performing well-designed, appropriately powered RCT in this space include but are not limited to patient heterogeneity with regard to the severity of the underlying disease or the stage of cirrhosis), poorly understood biological variability, lack of requirement by regulatory agencies for the performance of pivotal studies prior to broad application of combined technologies, and as a result low motivation by industry to support well-conducted, multicenter prospective RCTs. One single-center RCT comparing TACE + RFA to RFA alone was shown in a prospective RCT to improve local control and survival for patients with tumors measuring $3-5 \mathrm{~cm}$ but not for those $\leq 3 \mathrm{~cm}$ [6] likely due to the technical limitations of RFA for tumors $>3 \mathrm{~cm}$. Still, the relative therapeutic efficacy between TACE + PA versus TACE alone has not been prospectively studied for early-intermediate or recurrent unresectable $\mathrm{HCC} \leq 5 \mathrm{~cm}$. One such study showing a benefit of TACE + PA was published, but retracted due to concerns over its scientific validity $[7,8]$.

In this issue of Digestive Diseases and Sciences, Song et al. [9] report a study from two tertiary academic centers in China. Records from 1254 adult patients collected from 2007 to 2013 with a first recurrent HCC after resection (bias due to likely well-compensated liver disease) within Milan criteria, but declining salvage liver transplantation or repeat resection, who could have been candidates for percutaneous ablation, were retrospectively evaluated for treatment choice, of whom 63 patients received TACE and 96 patients underwent TACE plus RFA. The choice of therapy was non-random, and the authors stated that patient made the decision regarding single or combined therapy based on a description of the procedures, expected outcomes (presumably provided by the treating clinicians), expected adverse effects (again from the treating physicians) and costs. The authors stated that the TACE was superselective, utilizing oxaliplatin, epirubicin, iodized oil, and gelatin foam. Further technical details of the embolization were not reported. RFA was performed 10-28 days after TACE when utilized. Smaller tumors $(\leq 3 \mathrm{~cm})$ were singly ablated whereas larger tumors were sequentially ablated with a target of at least $0.5 \mathrm{~cm}$ margin, with needle tract ablation. Additional RFA was allowed if dynamic CT showed evidence of incomplete ablation within 1 week of the initial RFA. Imaging was obtained at 4 weeks and assessed by modified response evaluation criteria in solid tumors (mRECIST). Up to four additional TACE procedures were allowed, with most patients requiring at least 2 TACE cycles to obtain initial control. Patients were followed with imaging every 3 months to identify local, and distant intrahepatic and extrahepatic disease progression. Analysis was according to ITT with a significant fraction of patients requiring additional systemic or radiotherapy for disease progression. To evaluate the causal effect of treatment assignment on progression-free and overall survival, the investigators applied Cox models with inverse-probability of treatment weighting based on a propensity score derived from factors associated with the treatment assignment. This is a standard, but likely imperfect approach used in retrospective studies such as this to overcome the confounding biases related to non-random treatment assignment.

Patients included in this study were predominantly thin young men (median age 45, BMI 22) predominantly with HBV-related HCC, of which a minority were cirrhotic. There was a trend towards a longer post-resection disease-free time between the groups, longer in combination therapy, which could reflect the trend towards lesser histologic grade in the initial tumors. Consistent with the possible assignment of patients with less aggressive tumor biology to the combination arm was a lower frequency of AFP $>400$ in the combination arm. Unadjusted 1-, 3-, and 5-year OS rates for the TACE + RFA group were $82.3 \%, 42.7 \%$, and $16.5 \%$, respectively, and $75.9 \%, 30.7 \%$, and $11.3 \%$, respectively, for the TACE group. Nevertheless, after propensity weighting, differences in OS were not statistically significant. Unadjusted 
1-, 3-, and 5-year DFS rates were $55.1 \%, 22.5 \%$, and $9.7 \%$ in the TACE + RFA group, and $41.1 \%, 9.9 \%$, and $4.9 \%$ in the TACE group, respectively, a difference that remained significant after propensity weighting (hazard ratio 1.59; $95 \%$ CI 1.13-2.23; $P=0.007)$. Factors associated with improved DFS included initial BCLC stage and tumor size, cirrhosis and latency between resection and recurrence, likely a surrogate for biologically aggressive disease.

Based on the present data, can it be concluded that TACE + PA offers any advantage over TACE alone in patients with $\mathrm{HCC} \leq 5 \mathrm{~cm}$ ? I would argue that the answer remains "no" and that a well-conducted RCT is essential to answering this question. Some factors that limit generalizability of the authors' results include: (1) the population studied was extremely atypical for the general early-intermediate stage unresectable patient population usually treated with these modalities, being very young, noncirrhotic, and having already survived resection; (2) IPTW weighting did not likely completely balance the unmeasured confounder of tumor biology that might explain the improved DFS observed even after weighting; (3) the relevance of DFS and/or TTP rather than OS as an endpoint has been questioned by experts in the field [10], and after weighting there was no evidence of a survival benefit; and (4) in the present study, PA was utilized at a median 2 weeks after TE and was still associated with a significant increase in hepatic decompensation in the form of new onset ascites [9]. PA, when combined with TE is usually applied during same hospitalization, not delayed. Combining TE + PA during a single hospitalization has recently been associated with increased patient discomfort, longer hospital length of stay and a trend towards higher major complications [11].

Thus, strong evidence regarding the benefit of adding PA on TE in early-to-intermediate unresectable $\mathrm{HCC} \leq 5 \mathrm{~cm}$ remains lacking. While challenging, a well-designed RCT with a more typical patient population with cirrhosis, stratified by Child-Turcotte-Pugh score, tumor size ( $\leq 3 \mathrm{~cm}$ vs. $3-5 \mathrm{~cm}$ ), tumor differentiation, and pre-treatment AFP powered for a survival endpoint by ITT remains essential for defining the efficacy of combinatorial locoregional therapy.

\section{References}

1. Serper M, Taddei TH, Mehta R, et al. Association of provider specialty and multidisciplinary care with hepatocellular carcinoma treatment and mortality. Gastroenterology. 2017;152:1954-1964.

2. Lencioni RA, Allgaier HP, Cioni D, et al. Small hepatocellular carcinoma in cirrhosis: randomized comparison of radio-frequency thermal ablation versus percutaneous ethanol injection. Radiology. 2003;228:235-240.

3. Wang C, Wang H, Yang W, et al. Multicenter randomized controlled trial of percutaneous cryoablation versus radiofrequency ablation in hepatocellular carcinoma. Hepatology. 2015;61:1579-1590.

4. Rubinstein MM, Kaubisch A, Kinkhabwala M, Reinus J, Liu Q, Chuy JW. Bridging therapy effectiveness in the treatment of hepatocellular carcinoma prior to orthotopic liver transplantation. $J$ Gastrointest Oncol. 2017;8:1051-1055.

5. Llovet JM, Bruix J. Systematic review of randomized trials for unresectable hepatocellular carcinoma: chemoembolization improves survival. Hepatology. 2003;37:429-442.

6. Peng ZW, Zhang YJ, Chen MS, et al. Radiofrequency ablation with or without transcatheter arterial chemoembolization in the treatment of hepatocellular carcinoma: a prospective randomized trial. J Clin Oncol. 2013;31:426-432.

7. DeAngelis CD, Fontanarosa PB. Retraction: Cheng B-Q, et al. Chemoembolization combined with radiofrequency ablation for patients with hepatocellular carcinoma larger than $3 \mathrm{~cm}$ : a randomized controlled trial. JAMA. 2008;299:1669-1677.

8. DeAngelis CD, Fontanarosa PB. Retraction: Cheng B-Q, et al Chemoembolization combined with radiofrequency ablation for patients with hepatocellular carcinoma larger than $3 \mathrm{~cm}$ : a randomized controlled trial. JAMA. 2009;301:1931.

9. Song Q, Ren W, Fan L, et al. Long-term outcomes of transarterial chemoembolization combined with radiofrequency ablation versus transarterial chemoembolization alone for recurrent hepatocellular carcinoma after surgical resection. Dig Dis Sci. (Epub ahead of print). https://doi.org/10.1007/s10620-019-05733-0.

10. Bruix J, Reig M, Sangro B. Assessment of treatment efficacy in hepatocellular carcinoma: Response rate, delay in progression or none of them. J Hepatol. 2017;66:1114-1117.

11. Kim W, Cho SK, Shin SW, Hyun D, Lee MW, Rhim H. Combination therapy of transarterial chemoembolization (TACE) and radiofrequency ablation (RFA) for small hepatocellular carcinoma: comparison with TACE or RFA monotherapy. Abdom Radiol (NY). 2019;44:2283-2292.

Publisher's Note Springer Nature remains neutral with regard to jurisdictional claims in published maps and institutional affiliations.

\section{Compliance with Ethical Standards}

Conflict of interest Support to institution - Bayer Healthcare Inc. 